\title{
Infecção hospitalar e causas múltiplas de morte
}

\author{
Nosocomial infection and multiple causes of death
}

\author{
Ruth N.T. Turrini ${ }^{1}$, Augusto H. Santo ${ }^{2}$
}

\section{Resumo}

Objetivo: este estudo se propôs a avaliar a possibilidade de se obter informações de infecção hospitalar por meio da declaração de óbito.

Material e métodos: foi feita revisão de prontuários de crianças que faleceram com mais de 48 horas de internação num hospital pediátrico, para coleta de dados sobre as causas múltiplas de morte. Também foram revistas as declarações de óbito, para verificar sua qualidade de preenchimento. Os atestados de óbito foram refeitos segundo as informações do prontuário, utilizando-se a classificação suplementar de causas externas de lesões e envenenamentos da Classificação Internacional de Doenças - $9^{a}$ revisão, para codificar as infecções hospitalares e procedimentos associados.

Resultados: após refazer os atestados, observou-se concordância da causa básica de morte entre atestados originais e refeitos de 69,9\%, com índice de Kappa de 0,65. No atestado médico original, identificou-se somente uma infecção hospitalar, enquanto pelo atestado refeito recuperaram-se $88,9 \%$ de todas as infecções hospitalares, ocorridas nas crianças, até 15 dias antes do óbito.

Conclusão: a qualidade de preenchimento dos atestados de óbito não permite utilizar as declarações de óbito como fonte de dados de infecção hospitalar, a não ser que haja capacitação dos profissionais médicos para identificar e registrar as infecções hospitalares no prontuário, bem como preencher os atestados adequadamente.

J Pediatr (Rio J) 2002;78(6):485-90: infecção hospitalar, causa de morte, declaração de óbito, crianças.

\section{Introdução}

Os avanços tecnológicos relacionados aos procedimentos invasivos, diagnósticos e terapêuticos, e o aparecimento de microrganismos multirresistentes aos antimicrobianos usados rotineiramente na prática hospitalar tornaram as

1. Doutora em Saúde Pública. Professor Assistente do Dep. de Enfermagem Médico-Cirúrgica da Escola de Enfermagem da Universidade de São Paulo.

2. Orientador. Professor Associado do Dep. de Epidemiologia da Faculdade de Saúde Pública da Universidade de São Paulo.

Artigo submetido em 24.04.02, aceito em 14.08.02.

\begin{abstract}
Objective: the purpose of this study was to evaluate the possibility for collecting data on nosocomial infections by means of death certificates.

Methods: the medical charts of children who died after 48 hours of their admission to a pediatric hospital were revised to get information about the causes of death. Death certificates were also revised to verify whether they were properly filled out. The death certificates were redrafted according to the data obtained from patients' medical charts. Nosocomial infections and procedures related to them were codified using the Supplementary External Causes of Injury and Poisoning codes of the International Disease Classification - 9th Revision.
\end{abstract}

Results: the causes mentioned on medical death certificates compared with revised causes obtained after revision of patients' charts showed an agreement of $69.9 \%$, with a Kappa coefficient of 0.65 . Only one case of nosocomial infection was found on original death certificates, whereas the redrafted certificates revealed $88.9 \%$ of nosocomial infections in the study population up to fifteen days before death.

Conclusion: death certificates are not a good source of information on nosocomial infection because they are often filled out inadequately. The solution to this is to qualify physicians so that they can identify and record nosocomial infections on the patient's medical chart and complete death certificates accordingly.

J Pediatr (Rio J) 2002;78(6):485-90: nosocomial infection, cause of death, death certificate, children.

infecções hospitalares um problema de saúde pública. As maiores taxas de infecção hospitalar são observadas em pacientes nos extremos da idade e nos serviços de oncologia, cirurgia e terapia intensiva. Desta forma, os dados de incidência e prevalência de infecção hospitalar obtidos em diferentes estudos, mesmo em crianças, refletem tais características populacionais e institucionais ${ }^{1-3}$.

No Brasil, os dados sobre infecção hospitalar são pouco divulgados. Além disso, esses dados não são consolidados por muitos hospitais, o que dificulta o conhecimento da 
dimensão do problema no país. Como os pacientes que falecem após 48 horas de internação freqüentemente apresentam infecção hospitalar associada ${ }^{1}$, as causas de morte mencionadas no atestado médico da declaração de óbito se constituem em importante fonte de dados para o dimensionamento do problema. A Organização Mundial da Saúde (OMS) recomenda que as estatísticas de mortalidade sejam apresentadas segundo a denominada "causa básica de morte", definida como "(a) doença ou lesão que iniciou a cadeia de acontecimentos patológicos que conduziram diretamente à morte, ou (b) as circunstâncias do acidente, ou violência que produziram a lesão fatal". A causa básica de morte é apresentada nas chamadas estatísticas primárias, que tradicionalmente descrevem a mortalidade de populações. $\mathrm{O}$ controle de doenças que provocavam mortes precoces determinou o aumento da sobrevida de populações. Para descrever melhor o padrão da mortalidade nessas populações, houve necessidade de levar em consideração, além da causa básica, todas as demais mencionadas na declaração de óbito. Deste modo, as afecções geradas como complicações da causa básica, chamadas causas conseqüenciais, e outras presentes no momento da morte, chamadas causas contribuintes, somadas, definem as causas múltiplas de morte. Nos Estados Unidos, em 1988, a freqüência de infecções hospitalares, determinada por meio das múltiplas causas de morte, foi de 3,83/100.000 pessoas/ano ${ }^{4}$.

Limitações quantitativas e qualitativas comprometem respectivamente a cobertura e a fidedignidade dos dados sobre mortalidade. No Estado de São Paulo, a cobertura é praticamente total, não sendo influenciada pelo sub-registro de óbitos. Porém, o mesmo não se pode dizer da qualidade dos dados, desde que muitas variáveis da declaração de óbito apresentam valores significativos de dados ignorados ou em branco. A fidedignidade da causa básica da morte também é comprometida, quando a mesma diferir da conceituada pela OMS. Estes problemas estão relacionados ao conhecimento médico sobre o preenchimento adequado do atestado de óbito, e às informações incompletas disponíveis por ocasião da morte ${ }^{5}$. Estudos realizados para avaliar a fidedignidade da informação sobre a causa básica de morte encontraram concordância entre a causa inscrita no atestado de óbito original e a causa básica refeita por meio de dados complementares, oscilando entre 60,0 e $70,0 \%$ 6-8.

Este estudo tem por objetivo verificar a qualidade de preenchimento dos atestados de óbito e a presença do diagnóstico de infecção hospitalar como causa de morte nas declarações de óbito de pacientes que evoluíram a óbito em um hospital pediátrico.

\section{Metodologia}

A população de estudo foi constituída por crianças que faleceram após 48 horas de internação, num hospital público pediátrico de atenção terciária à saúde, no município de São Paulo, no ano de 1993. Na escolha do ano de ocorrência dos óbitos, considerou-se a dinâmica institucional de revisão dos prontuários de pacientes falecidos, que precede a inserção de dados no sistema informatizado, para que se obtivesse a relação exata dos óbitos ocorridos.

A lista dos óbitos foi obtida junto ao serviço de processamento de dados do estado de São Paulo, a partir da qual recuperaram-se os prontuários dos pacientes e, posteriormente, as declarações de óbito correspondentes junto ao Programa de Aprimoramento das Informações de Mortalidade, no município de São Paulo (PROAIM), e na Fundação Sistema Estadual de Análises de Dados (SEADE). As causas de morte foram codificadas segundo a Classificação Internacional de Doenças, $9^{a}$ Revisão (CID-9) ${ }^{9}$.

O preenchimento do atestado médico da declaração de óbito foi feito no próprio hospital de internação, pelo médico responsável, ou plantonista, nos casos de óbitos bem definidos. A declaração de óbito foi fornecida pelo Serviço de Verificação de Óbitos da Faculdade de Medicina da Universidade de São Paulo, para os óbitos mal definidos, e pelo Instituto Médico Legal nas mortes devidas a causas não naturais. Os prontuários foram revistos pelo primeiro autor (RNTT). Em caso de dúvida, o prontuário foi reavaliado por um médico. O segundo autor (AHS), especialista na área de codificação de causas de morte, reviu os atestados de óbito quanto à adequação de seu preenchimento.

As causas de morte mencionadas no atestado médico da declaração de óbito original foram analisadas e comparadas com as causas de morte que foram refeitas a partir da revisão dos prontuários dos pacientes falecidos. Além das causas básicas de morte, também foram consideradas as demais causas associadas, isto é, as causas conseqüenciais e as contribuintes, integradas como causas múltiplas de morte, e que foram processadas pelo Tabulador de Causas Múltiplas de Morte $^{10}$. No atestado de óbito refeito, as infecções hospitalares foram identificadas no agrupamento das complicações de cuidados cirúrgicos e médicos (996999), do Capítulo 17 (Lesões e Envenenamentos), da CID9, por meio dos seguintes códigos: peritonite 996.6, ventriculite 997.0, endocardite 997.1, flebite 997.2, pneumonia 997.3, diarréia e enterocolite necrosante 997.4, infecção urinária 997.5, infecção pós-operatória 998.5, e outras infecções (pele ou infecção de corrente sanguínea) 999.3. Os procedimentos médicos foram identificados por meio de rubricas específicas da Classificação Suplementar de Causas Externas de Lesões e de Envenenamentos da CID-99. Para as cirurgias associadas à infecção, foram utilizados códigos da categoria "reação anormal em paciente, ou complicação tardia, causada por intervenção cirúrgica ou por outros atos cirúrgicos, sem menção de acidente ao tempo da intervenção (E878)". Para os demais procedimentos, foi usada a categoria "reação anormal em paciente ou complicação tardia, causada por outros procedimentos, sem menção de acidente ao tempo do procedimento (E879)". O cateterismo urinário recebeu o código E879.6, e os procedimentos relacionados à ventilação mecânica, cateter 
central ou diálise peritoneal foram codificadas em E879.8. As infecções hospitalares, ocorridas nos últimos quinze dias que antecederam o óbito, foram recuperadas dos dados registrados nos prontuários dos pacientes falecidos, e caracterizadas segundo os critérios elaborados pelo Centers for Diseases Control e usados pelo National Nosocomial Infection Surveillance System ${ }^{11}$.

A qualidade das informações sobre a causa básica de morte foi aferida por meio do índice Kappa ${ }^{12}$ e cálculo de sensibilidade, especificidade e valores preditivos positivo e negativo ${ }^{13}$.

\section{Resultados}

Os óbitos estudados (140) representaram $50,4 \%$ de todos os óbitos ocorridos no hospital, e $92,5 \%$ dentre os falecidos após de 48 horas de internação. O coeficiente geral de mortalidade hospitalar foi de 7 óbitos por 1.000 pacientes-dia, e o de mortalidade com mais de 48 horas de internação foi de 4 óbitos por 1.000 pacientes-dia. Foram considerados bem definidos 46 (32,6\%) óbitos, mal definidos $88(62,4 \%)$, e sete $(5,0 \%)$ relacionados a crianças institucionalizadas, ou a mortes violentas. Os óbitos mal definidos correspondem àqueles encaminhados ao Serviço de Verificação de Óbitos, para realização de autópsia, independentemente da causa de morte. Quatro crianças institucionalizadas provenientes da FEBEM, uma criança com traumatismo craniencefálico por queda, um grande queimado por incêndio de carro e um recém-nascido com asfixia neonatal grave por intoxicação exógena tiveram a declaração de óbito preenchida no Instituto Médico-Legal. Quatro declarações de óbito não foram localizadas e, portanto, a análise restringiu-se a 136 óbitos.

Na declaração de óbito, há campos designados à obtenção de informações sobre a afecção do paciente. Aquele relativo ao intervalo de tempo entre o início do processo mórbido e o óbito não foi preenchido nas 136 declarações de óbito localizadas; o item sobre realização de exame complementar foi preenchido em $26(19,1 \%)$; o item para realização de necropsia em 99 (72,8\%); e o item sobre intervenção cirúrgica em 16(11,8\%). Verificou-se que esse último item havia sido preenchido em apenas $3(13,0 \%)$ dos 23 pacientes submetidos a algum procedimento cirúrgico.

A Tabela 1 mostra a distribuição das causas de morte, segundo os capítulos da CID-9, informadas nos atestados de óbito original e refeito, e a concordância entre os mesmos e respectivos testes de validade. Verifica-se que as quatro principais causas de morte, anomalias congênitas, doenças do aparelho respiratório, afecções originadas no período perinatal e neoplasias, permaneceram ordenadas na mesma sequiência em ambos os atestados, original e refeito, embora com valores diferentes. As doenças infecciosas e parasitárias passaram da sétima para a quinta posição. O maior excesso de causas informadas no atestado original estavam incluídas nas doenças do aparelho respiratório.

Tabela 1 - Teste de validação das causas básicas de morte declaradas em relação às causas básicas de morte, refeitas a partir dos prontuários dos pacientes, Hospital Pediátrico Terciário, São Paulo, 1993

\begin{tabular}{|c|c|c|c|c|c|c|c|c|}
\hline Capítulo da CID & $\begin{array}{l}\text { Causa básica } \\
\text { refeita }\end{array}$ & $\begin{array}{c}\text { Causa básica } \\
\text { original }\end{array}$ & $\begin{array}{c}\text { Concor- } \\
\text { dância }\end{array}$ & $\begin{array}{l}\text { VPP } \\
\%\end{array}$ & $\begin{array}{c}\text { VPN } \\
\%\end{array}$ & $\begin{array}{l}\text { Sensibi- } \\
\text { lidade \% }\end{array}$ & $\begin{array}{l}\text { Especifi- } \\
\text { cidade \% }\end{array}$ & $\begin{array}{l}\text { Índice } \\
\text { Kappa }\end{array}$ \\
\hline Doenças infecciosas e parasitárias & 12 & 10 & 5 & 50,0 & 94,4 & 41,7 & 96,0 & 0,41 \\
\hline Neoplasias & 15 & 14 & 14 & 100,0 & 99,2 & 93,3 & 100,0 & 0,96 \\
\hline $\begin{array}{l}\text { Doenças das glândulas endócrinas, } \\
\text { da nutrição e do metabolismo e ... }\end{array}$ & 11 & 12 & 8 & 66,7 & 97,6 & 72,7 & 96,8 & 0,67 \\
\hline $\begin{array}{l}\text { Doenças do sistema nervoso e dos órgãos } \\
\text { dos sentidos }\end{array}$ & 8 & 6 & 4 & 66,7 & 96,9 & 50,0 & 98,4 & 0,55 \\
\hline Doenças do aparelho circulatório & 5 & 4 & 4 & 100,0 & 99,2 & 80,0 & 100,0 & 0,89 \\
\hline Doenças do aparelho respiratório & 19 & 25 & 12 & 48,0 & 93,7 & 63,1 & 88,9 & 0,46 \\
\hline Doenças do aparelho digestivo & 9 & 13 & 5 & 38,5 & 96,7 & 55,5 & 93,7 & 0,41 \\
\hline Doenças do aparelho geniturinário & 1 & - & - & - & 99,3 & - & 100,0 & - \\
\hline $\begin{array}{l}\text { Doenças da pele e do tecido } \\
\text { celular subcutâneo }\end{array}$ & 1 & - & - & - & 99,3 & - & 100,0 & - \\
\hline $\begin{array}{l}\text { Doenças do sistema osteomuscular } \\
\text { e do tecido conjuntivo }\end{array}$ & 1 & - & - & - & 99,3 & - & 100,0 & - \\
\hline Anomalias congênitas & 34 & 32 & 27 & 84,4 & 93,3 & 79,4 & 95,1 & 0,76 \\
\hline Algumas afecções do período perinatal & 18 & 16 & 15 & 93,8 & 97,5 & 83,3 & 99,1 & 0,87 \\
\hline Sintomas, sinais e afecções mal definidas & - & 2 & - & - & 100,0 & - & 98,5 & - \\
\hline Causas externas & 2 & 2 & 1 & 50,0 & 99,3 & 50,0 & 99,2 & 0,49 \\
\hline Total & 136 & 136 & 95 & & & & & \\
\hline
\end{tabular}

VPP: valor preditivo positivo 
A Tabela 2 mostra as causas associadas de morte observadas, respectivamente, nos atestados refeito e original, relativas aos 136 óbitos estudados. O fato relevante verificado refere-se à identificação e inclusão dos procedimentos médico-cirúrgicos no atestado refeito. Conseqüentemente, as complicações desses procedimentos, geralmente afecções terminais associadas, que no atestado original receberam códigos incluídos em capítulos das doenças, passaram a ser codificadas como complicações pós-procedimentos, neste estudo caracterizadas como infecções hospitalares. Como exemplo, pneumonias classificadas nas doenças do aparelho respiratório, no atestado refeito, foram caracterizadas como pneumonias pós-procedimentos e codificadas em rubrica incluída no agrupamento das complicações de cuidados médicos e cirúrgicos. Além disso, em ambos os atestados, original e refeito, nota-se que os sinais, sintomas e afecções mal definidas apresentam o maior número de causas associadas, dado que os diagnósticos incluídos nesse capítulo são mencionados como causas terminais com muita freqüência.

No atestado original, foram mencionadas cinco intervenções cirúrgicas e, no atestado refeito, sete, além de 24 outros procedimentos médicos. Estes procedimentos referiram-se ao uso de ventilação mecânica, cateter de diálise peritoneal, cateter vesical e cateter venoso, e representaram $57,1 \%$ de todos os procedimentos realizados nas crianças estudadas.

Ao se refazer o atestado de óbito, incluindo-se as informações do prontuário relacionadas às infecções hospitalares ocorridas até quinze dias do óbito, desde que associadas ao evento morte como causa conseqüencial, verificou-se que as infecções hospitalares representaram 88,9\% das infecções ocorridas nesse período. Pelo atestado médico original, detectou-se apenas uma infecção hospitalar.

De modo geral, observou-se que em 69,9\% dos atestados médicos originais, a causa básica de morte registrada coincidiu com aquela dos atestados refeitos. As doenças por capítulo da CID-9, com os maiores valores preditivos positivos, foram as neoplasias $(100,0 \%)$, as doenças do aparelho circulatório $(100,0 \%)$, as afecções originadas no período perinatal $(93,8 \%)$ e as anomalias congênitas $(84,4 \%)$. As afecções registradas de maior sensibilidade pertenciam ao capítulo das neoplasias $(93,3 \%)$, das doenças do aparelho circulatório $(80,0 \%)$ e das afecções originadas no período perinatal $(83,3 \%)$. Por esses dados, constata-se que os melhores atestados são aqueles com causa básica de morte codificada no capítulo das neoplasias, doenças do aparelho circulatório, afecções do período perinatal e anomalias congênitas.

Utilizando-se o índice Kappa para comparar a qualidade de preenchimento entre o atestado de óbito original e o refeito, através das causas básicas agrupadas por capítulo da CID-9, obteve-se índice de 0,65, IC95\% [0,57; 0,74].

Individualmente, por capítulos da CID-9, o índice Kappa foi superior a 0,80 nas doenças cardiovasculares,
Tabela 2 - Causas associadas de morte, segundo capítulos e categorias de infecção hospitalar - CID-9, Hospital Pediátrico Terciário, São Paulo, 1993

\begin{tabular}{lcc}
\hline Causas associadas de morte & $\begin{array}{c}\text { Atestado } \\
\text { refeito }\end{array}$ & $\begin{array}{c}\text { Atestado } \\
\text { original }\end{array}$ \\
\hline Doenças infecciosas e parasitárias & 25 & 31 \\
Neoplasias & - & 2 \\
Doenças das glândulas endócrinas, & 16 & 8 \\
da nutrição ... & & \\
Doenças do sangue e dos órgãos & 4 & 6 \\
hematopoiéticos & & \\
Doenças do sistema nervoso e dos órgãos & 12 & 15 \\
dos sentidos & 12 & 11 \\
Doenças do aparelho circulatório & 23 & 43 \\
Doenças do aparelho respiratório & 24 & 20 \\
Doenças do aparelho digestivo & 14 & 9 \\
Doenças do aparelho geniturinário & & \\
Doenças da pele e do tecido & - & 2 \\
celular subcutâneo & 9 & 12 \\
Anomalias congênitas & & \\
Algumas afecções originadas & 194 & 194 \\
no período perinatal & 4 & 4 \\
Sintomas, sinais e afecções mal definidas & 62 & 50 \\
Lesões e envenenamentos NCOP & 2 & 2 \\
Peritonite pós-procedimento & 2 & - \\
Ventriculite pós-procedimento & 1 & - \\
Endocardite pós-procedimento & 1 & - \\
Flebite pós-procedimento & 1 & - \\
Pneumonia pós-procedimento & 25 & - \\
Diarréia pós-procedimento & 6 & - \\
Infecção urinária pós-procedimento & 8 & - \\
Infecção pós-operatória & 3 & 1 \\
Outras infecções pós-procedimento & 33 & - \\
Causas externas NCOP & 6 & 4 \\
Procedimento cirúrgico & 7 & 5 \\
Outros procedimentos médicos & 24 & - \\
\hline Total & & \\
\hline & & \\
\hline
\end{tabular}

neoplasias e afecções do período perinatal. A especificidade foi acima de 80,0\% para todos os capítulos estudados, enquanto a sensibilidade acima de 80,0\% acompanhou os índices Kappa (Tabela 1).

\section{Discussão}

Apesar do modelo uniforme de declaração de óbito, adotado pelo Ministério da Saúde em 1976, e das definições precisas de causa básica de mortalidade, as estatísticas de mortalidade ainda não são inteiramente corretas ${ }^{14}$. Isto se observa nas publicações sobre a qualidade das informações contidas no atestado de óbito. 
Estudo sobre a qualidade da certificação médica da causa de morte em São Paulo, em 1971 e 1972, evidenciou que $93,5 \%$ dos casos não apresentavam o preenchimento do item intervalo entre o início do processo mórbido e o óbito e, em relação ao item exame complementar, $68,0 \%$ não continham essa informação. Além disso, $31,4 \%$ dos atestados não apresentavam a verdadeira causa de morte declarada, e, em $6,5 \%$, a mesma estava declarada, porém não foi selecionada como causa básica após a aplicação das regras de mortalidade da CID-9 ${ }^{15}$.

Neste estudo, houve exatidão em relação à codificação da causa básica de morte em quase $70,0 \%$ dos casos, dado este próximo aos citados pela literatura, nos quais as concordâncias giram em torno de 60,0 a $70,0 \%{ }^{5,14,15}$. Os resultados revelaram que as doenças do trato respiratório foram as mais superdiagnosticadas. Resultado semelhante foi observado em outro estudo ${ }^{6}$, e os autores apontam como uma das razões para as doenças respiratórias serem superestimadas o fato delas surgirem no estágio final da doença e, assim, o médico as considera como causa básica, não levando em conta o quadro clínico que deu início ao processo.

Como o hospital do presente estudo é de referência terciária, justifica-se o maior número de óbitos por neoplasias e outras afecções de alto grau de complexidade, conferindo-lhe perfil de mortalidade diferente de outras citações bibliográficas. Uma investigação para estudar a fidedignidade dos atestados de óbito de crianças que evoluíram a óbito, na unidade infantil para menores de 15 anos da Fundação Hospitalar de Santa Catarina, por meio da revisão de prontuários, identificou como causas mais freqüentes de óbito as doenças infecciosas e parasitárias $(21,6 \%)$, as anomalias congênitas $(15,2 \%)$ e as afecções originadas no período perinatal $(15,2 \%)^{14}$.

Segundo a classificação de Morgenstern ${ }^{16}$, o índice Kappa mostrou boa concordância e foi semelhante a de outros autores, conforme o grupo populacional e a causa básica estudados ${ }^{6}$. No entanto, diferem do estudo realizado no Rio Grande do Sul, em crianças maiores de sete dias e menores de 364 dias, para comparar as causas de óbito no atestado original com o refeito, em que observaram-se valores de concordância menores, como no caso das doenças infecciosas e desnutrição ${ }^{17}$.

Observando-se a semelhança entre os resultados do presente estudo e de outros $6,7,14,15$, pode-se supor que a confiabilidade dos dados das causas básicas de morte infantis declaradas é semelhante nas regiões metropolitanas da região sudeste do Brasil. Estudo que utilizou como fonte de dados os resultados de necropsias para medir a concordância da causa de morte com o atestado de óbito evidenciou concordância por categoria de doenças da CID-9 de $71,0 \%$, quase igual à deste estudo em crianças. No entanto, na análise mais detalhada, identificaram-se pequenas diferenças que provavelmente refletem o perfil da população investigada. As categorias de maior valor preditivo positivo foram as causas externas, neoplasias e afecções digestivas, aquelas de maior sensibilidade foram dos sinais, sintomas e afecções mal definidas, anomalias congênitas e neoplasias. As doenças do sistema circulatório foram as mais superdiagnosticadas no atestado de óbito, e as doenças gastrintestinais as mais subdiagnosticadas ${ }^{5}$.

Em um estudo comparativo de causas múltiplas de morte dos óbitos ocorridos em 1955 e 1980, na Califórnia, os autores concluíram que a utilização das causas múltiplas tem algumas vantagens sobre a causa única de morte: permitir estudo combinado da doença com fatores que aumentam a mortalidade, compreender o papel das doenças não fatais como contribuintes para a morte e responder perguntas sobre o aumento, ou declínio, das causas de morte ${ }^{18}$. Se os atestados de óbito fossem claramente preenchidos, as infecções hospitalares contribuintes ao óbito poderiam ser identificadas e gerar informações sobre a freqüência desse evento, bem como dimensionar o problema.

Neste estudo, quando as causas associadas das lesões e envenenamentos, que incluem as infecções hospitalares, foram desmembradas nas subcategorias, obteve-se maior número de causas associadas. Isto ocorreu porque, quando os múltiplos diagnósticos são reunidos em classes de maior amplitude, as doenças perdem a individualidade e adquirem o significado das classes mais amplas ${ }^{19}$.

Constatou-se, ainda, que somente informações sobre as infecções hospitalares ocorridas nas cirurgias podem ser obtidas por este método de coleta de dados, a partir das declarações de óbito e, mesmo assim, em menor número do que quando o atestado de óbito foi refeito. Além disto, os dados presentes nos atestados de óbito não são suficientes para determinar se a infecção hospitalar foi contribuinte ao óbito.

Nos Estados Unidos, White ${ }^{4}$ encontrou proporção de pneumonias entre os óbitos de $55,0 \%$, valor maior do que aquele obtido pelos dados de morbidade da vigilância epidemiológica, porém semelhante ao estudo de Gross et al. ${ }^{20}$, em que $60,0 \%$ dos pacientes que morreram com infecção hospitalar tinham pneumonia na revisão de prontuários.

Pelas observações feitas após o repreenchimento dos atestados de óbito, segundo informações do prontuário, percebeu-se que quase 90,0\% das infecções hospitalares contribuintes, ou causa imediata do óbito, poderiam ter sido identificadas por este método de coleta, caso o preenchimento fosse adequado.

Para que a declaração de óbito possa ser utilizada como fonte de dados de infecção hospitalar associada ao óbito, é imprescindível a melhora na qualidade de preenchimento dos atestados de óbito. O campo sobre tempo de início da doença deve ser sempre preenchido. Além disto, nos atestados de óbito, é preciso que se introduza um campo para discriminar a infecção hospitalar e outro para procedimentos associados, já que existe atualmente apenas o campo para cirurgias. 
Não é suficiente a criação de um novo modelo de declaração de óbito, se não houver conscientização por parte dos profissionais da saúde sobre a importância de se informar a presença de infecções hospitalares.

Se os dados de infecção hospitalar pudessem ser obtidos por meio de um banco de dados de morbimortalidade, ou pelos atestados de óbitos, poder-se-ia avaliar melhor a dimensão do problema em nível nacional.

Sugere-se capacitação dos profissionais médicos, de modo a habilitá-los a preencherem adequadamente os atestados médicos e, desta forma, viabilizar a utilização das declarações de óbito como fonte de dados de infecção hospitalar, como causa imediata ou contribuintes ao óbito.

\section{Referências bibliográficas}

1. Turrini RNT. Infecção hospitalar e mortalidade em hospital pediátrico [dissertação]. São Paulo: Faculdade de Saúde Pública da Universidade de São Paulo;1996.

2. Avila-Figueroa C, Casta-Cruz M, Aranda-Patrón E, León AR, Justiniani N, Perez-Ricardez L, et al. Prevalencia de infecciones nosocomiales en niños: encuesta de 21 hospitales en Mexico. Salud Publica Mex 1999;41:528-5.

3. Gilio AE, Stape A, Pereira CR, Cardoso MF, Silva CV, Troster EJ, et al. Risk factors for nosocomial infections in a critically ill pediatric population: a 25 -month prospective cohort study. Inf Control Hosp Epidemiol 2000;21:340-2.

4. White MC. Mortality associated with nosocomial infections: analysis of multiple cause-of-death. J Clin Epidemiol 1993;46: 95-100.

5. Kircher T, Nelson J, Burdo H. The autopsy as a measure of accuracy of the death certificate. N Engl J Med 1985;313:1263-9.

6. Carvalho ML, Niobey FML, Miranda NN, Sabroza PC. Concordância na determinação da causa básica de óbito em menores de um ano na região metropolitana do Rio de Janeiro, 1986. Rev Saúde Pública 1990;24:20-7.

7. Mendonça EF, Goulart MA, Machado JAD. Confiabilidade da declaração de causa básica de mortes infantis em região metropolitana do sudeste do Brasil. Rev Saúde Pública 1994; 28:385-91.

8. Niobey FML, Cascão AM, Duchiade MP, Sabroza PC. Qualidade do preenchimento de atestados de óbitos de menores de um ano na região metropolitana do Rio de Janeiro. Rev Saúde Pública 1990;24:311-8.
9. Organização Mundial da Saúde. Manual de classificação estatística internacional de doenças, lesões e causas de óbito; $9^{\text {a }}$ Revisão, 1975. São Paulo, Centro da OMS para Classificação de Doenças em Português; 1978.v.1.

10. Santo AH, Pinheiro CE. Tabulador de causas múltiplas de morte. Rev Brasil Epidemiol 1999;2:90-9.

11. Centers for Disease Control. National nosocomial infection surveillance (NNIS). Atlanta; 1994.

12. Cohen J. A coefficient of agreement for nominal scales. Educ Psychol Measurem 1960;20:37-46.

13. Kleinbaum DG, Kupper LL, Morgenstern H. Epidemiologic research: principles and quantitative methods. New York: John Wiley \& Sons; 1982.

14. Strozzi GM, Strozzi JB, Souza ML, Schutel MD. Estudo de causa básica de óbitos de menores de 15 anos ocorridos em hospital de Florianópolis, Santa Catarina (Brasil), em 1982. Rev Saúde Pública 1985;19:123-32.

15. Fonseca LA, Laurenti R. A qualidade da certificação médica da causa de morte em São Paulo, Brasil. Rev Saúde Pública 1974;8:21-9.

16. Morgenster H. apud Monteiro GTR. Validação diagnóstica dos atestados de óbitos com neoplasia como causa básica no Estado do Rio de Janeiro [tese]. Rio de Janeiro: Escola Nacional de Saúde Pública; 1995.

17. Nobre LC, Victoria CG, Barros FC, Lombardi C, Teixeira AMB, Fuchs SC. Avaliação da qualidade da informação sobre a causa básica de óbitos infantis no Rio Grande do Sul (Brasil). Rev Saúde Pública 1989;23:207-13.

18. White MC, Selvin S, Merrill DW. A study of multiple cause of death in California: 1955 and 1980. J Clin Epidemiol 1989; 42:355-65.

19. Santo AH. Causas múltiplas de morte: formas de apresentação e métodos de análise [tese]. São Paulo: Faculdade de Saúde Pública da Universidade de São Paulo; 1988.

20. Gross PA, Neu CC, Aswapokee P, Van Antwerpen C, Aswapokee N. Deaths from nosocomial infections: experience in a University Hospital and a Community Hospital. Am J Med 1980;68:219-23.

\author{
Endereço para correspondência: \\ Dra. Ruth N.T. Turrini \\ Rua Xavier de Almeida, 743 \\ CEP 04211-001 - São Paulo, SP \\ Fone: (11) 3066.7558 \\ E-mail: rturrini@usp.br
}

